\title{
CORRIGENDUM
}

\section{Landau fluid closures with nonlinear large-scale finite Larmor radius corrections for collisionless plasmas - CORRIGENDUM}

\section{P. L. Sulem and T. Passot}

doi: 10.1017/S0022377814000671, Published by Cambridge University Press, 5 September 2014.

The authors would like to correct several misprints in the original article (Sulem and Passot 2014).

In Eq. (3.7), $\boldsymbol{\omega}_{p}=\nabla \wedge \boldsymbol{u}_{p}$ denotes the proton vorticity.

In the second line of Eq. (3.23), $\boldsymbol{n} \cdot \Pi$ should be replaced by $\boldsymbol{b} \cdot \Pi$.

Equation (5.27) should read

$$
\Pi_{\perp i j}^{S S}=\left(n_{i p} n_{j q}-r_{i p} r_{j q}\right) \partial_{p} \partial_{q}\left(-\Delta^{-1} \mathscr{A}^{S S}\right)-\left(n_{i p} r_{j q}+n_{j p} r_{i q}\right) \partial_{p} \partial_{q}\left(-\Delta^{-1} \mathscr{B}^{S S}\right) .
$$

Equation (D 4) should read

$$
\Pi_{\perp}=-\left[\nabla_{\perp} \otimes \nabla_{\perp}-(\boldsymbol{b} \wedge \nabla) \otimes(b \wedge \nabla)\right] \Delta_{\perp}^{-1} \mathscr{A}-\left[\nabla_{\perp} \otimes(b \wedge \nabla)+(b \wedge \nabla) \otimes \nabla_{\perp}\right] \Delta_{\perp}^{-1} \mathscr{B},
$$

and Eq. (D 5)

$$
\Pi_{\perp i, j}=\left(n_{i p} n_{j q}-r_{i p} r_{j q}\right) \partial_{p} \partial_{q}\left(-\Delta_{\perp}^{-1} \mathscr{A}\right)-\left(n_{i p} r_{j q}+n_{j p} r_{i q}\right) \partial_{p} \partial_{q}\left(-\Delta_{\perp}^{-1} \mathscr{B}\right) .
$$

Table 1 should read as below:

$$
\begin{array}{ll}
\text { Bessel coefficients } & \text { behavior for } b \rightarrow 0 \\
\mathfrak{A}_{1}=1-\Gamma_{1} / \Lambda_{2}-\mathfrak{A}_{2} & 1 / 2+b / 4 \\
\mathfrak{E}_{1} & 9 b / 4 \\
\mathfrak{E}_{4} & 9 / 4 \\
\mathfrak{E}_{6} & 3 \\
\mathfrak{H}_{3}=\left(\Gamma_{1}-2\right) / \Gamma_{0}+1 & -1-3 b / 2 \\
\mathfrak{H}_{4}=\Lambda_{3} / \Lambda_{2}+\mathfrak{H}_{3} & -3 b / 4 \\
\mathfrak{R}_{\perp}^{[2]}=-\mathfrak{R}_{\perp}^{[3]}+\left(\mathfrak{R}_{2} \Lambda_{3}-\mathfrak{R}_{3} \Gamma_{0}\right) / \Lambda_{2} & 9 b / 2 \\
\mathfrak{T}_{1}=-\Lambda_{3}+\mathfrak{E}_{1}-b \mathfrak{B}_{1} & 3 b / 2 \\
\mathfrak{T}_{2}=\mathfrak{T}_{1}+1 & 1+3 b / 2 \\
\mathfrak{Z}_{1} & -1-3 b / 2 \\
\mathfrak{Z}_{2} & -3 b / 4 \\
&
\end{array}
$$

